\title{
Site-Directed Mutagenesis by Double Polymerase Chain Reaction: Megaprimer Method
}

\author{
Sailen Barik \\ Cleveland State University, s.barik@csuohio.edu
}

Follow this and additional works at: https://engagedscholarship.csuohio.edu/scibges_facpub

Part of the Molecular Biology Commons

How does access to this work benefit you? Let us know!

\section{Publisher's Statement}

(c) 1993 Humana Press

\section{Recommended Citation}

Barik, S. (1993). Site-Directed Mutagenesis by Double Polymerase Chain Reaction: Megaprimer Method. In PCR protocols : current methods and applications, B.A. White, ed. Humana Press. p. 277-286.

This Contribution to Books is brought to you for free and open access by the Biological, Geological, and Environmental Sciences Department at EngagedScholarship@CSU. It has been accepted for inclusion in Biological, Geological, and Environmental Faculty Publications by an authorized administrator of EngagedScholarship@CSU.

For more information, please contact library.es@csuohio.edu. 


\title{
CHAPTER 28
}

\section{Site-Directed Mutagenesis by Double Polymerase Chain Reaction}

\author{
Megaprimer Method
}

\section{Sailen Barik}

\section{Introduction}

The "megaprimer" method (1) based on polymerase chain reaction (PCR) is one of the simplest and most versatile procedures of sitespecific in vitro mutagenesis available to date. The method utilizes three oligonucleotide primers and two rounds of PCR performed on a DNA template containing the cloned gene that is to be mutated. The rationale of the method is shown schematically in Fig. 1 where A and B represent the "flanking" primers that can map either within the cloned gene or outside the gene (i.e., within the vector sequence) and $M$ represents the internal "mutant" primer containing the desired base change. The first round of PCR is performed using the mutant primer (e.g., M1 in Fig. 1) and one of the flanking primers (e.g., A). The doublestranded product (A-M1) is purified and used as one of the primers (hence the name "megaprimer") in the second round of PCR along with the other flanking primer (B). The wild type cloned gene is used as template in both PCR reactions. The final PCR product (A-M1-B) containing the mutation can be used in a variety of standard applications, such as cloning in expression vectors and sequencing, or in more specialized applications, such as production of the gene message in vitro if primer $A$ (or the template sequence downstream of primer A) also contains a transcriptional promoter (e.g., that of SP6 or T7 phage). 


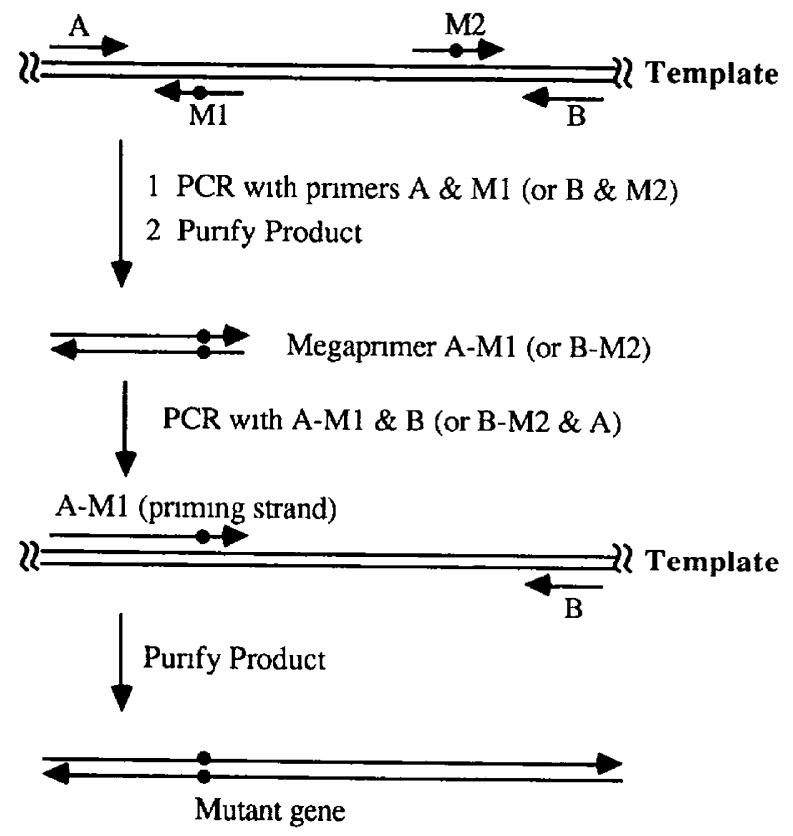

Fig. 1. The megaprimer method of site-specific mutagenesis. The primers A, B, $M 1$, and M2 (as well as the priming strand of the megaprimer, AM1) are indicated by single lines with arrowhead, whereas the double lines represent the template. The dot in M1 and M2 indicates the desired mutations (base changes) to be introduced into the product via the megaprimer. See text for details.

Both primers A and B are usually designed to contain convenient restriction sites so that the final, mutant PCR product can be restricted and cloned. When a gene is cloned within two unique sites (a multicloning site, for example) of a vector, primers A and B can be made at these sites. The mutant product can then be restricted and ligated at the same two sites in the same vector, and the final clone will retain the original flanking sequences of the gene. This is especially important when sequences upstream of A (e.g., a Shine-Dalgarno sequence or an upstream activation sequence, UAS) or downstream of B (e.g., a polyadenylation site or an RNase processing site) are essential for gene expression or regulation and must remain unaltered.

More often than not, the gene in question codes for a protein, and studies of structure-function relationship require the generation of a battery of mutant proteins altered at specific amino acid residues. In 
such cases, primers $A$ and $B$ can be kept constant and a variety of mutant primers (M1, M2, and so on) can be used to produce the various mutants. The reciprocal combination of primers (i.e., M2 and B in the first round of PCR; then A and megaprimer M2-B in the second round) can also be used provided primer M2 is of the opposite sense of $B$ (Fig. 1). Choice of primer location is described in more detail under Methods.

Since the megaprimer can be quite large (it may approach the size of the whole gene) and incorporated internal to the gene, successful and error-free PCR in the second round often requires special considerations. The reader is therefore strongly urged to go through the whole chapter, including the Notes section before proceeding with the actual experiment.

Note that the double-stranded megaprimer is directly used in the second round of PCR; prior separation of the two strands being unnecessary. Melting of the megaprimer is essentially achieved in the denaturation step of the PCR cycle itself. Although both strands of the megaprimer will anneal to the respective, complementary strands of the template, the basic rules of PCR amplification automatically ensures that only the correct strand (one that extends to the other primer, B, in Fig. 1) will be amplified into the double-stranded product. Under some conditions, particularly with large megaprimers $(\geq 1 \mathrm{~kb})$, self-annealing of the megaprimer tends to reduce the yield of the product (2). In order to avoid this, use of higher amounts of template (in microgram range, as opposed to nanogram quantities used in standard PCR) in the second PCR has been recommended in the method; this improves the final product yield in many cases (2), presumably because extra template strands anneal to both strands of the megaprimer, thereby preventing renaturation of the megaprimer.

\section{Materials}

1. DNA template containing the cloned gene (e.g., in pUC or pGEM vector) to be mutated.

2. Oligonucleotide primers A and B: the "upstream" primer A in the message sense and the "downstream" primer B in the antimessage sense. Include restriction sites, preferably unique, in these primers so that the final product can be restricted and cloned (see also Section 3.1. and Note 1).

3. Reagents for PCR (see Chapter 1).

4. A system for purifying the PCR products. An electroelutor (IBI, New Haven, CT) is used here. See Index for other methods in this volume. 
5. Reagents for agarose gel electrophoresis (see Chapter 1).

6. $8 M$ Ammonium acetate, $0.01 \%$ bromophenol blue.

7. TE buffer: $10 \mathrm{~m} M$ Tris- $\mathrm{HCl}, \mathrm{pH} 7.5,1 \mathrm{~m} M$ EDTA.

8. $\mathrm{CHCl}_{3}$.

9. TE-saturated phenol.

10. $95 \%$ Ethanol.

11. $70 \%$ Ethanol.

\section{Method \\ 3.1. Primer Design}

1. For technical reasons described here, avoid making megaprimers that approach the size of the final, full-length product (gene) AB (Fig. 1). If M1 is too close to B, it will make separation of AB and AM1 (leftover megaprimer) difficult after the second round of PCR. Ideally, the megaprimer should be shorter than the full-length gene by more than 200-500 bp, depending on the exact length of the gene. (Example: If the gene $\mathrm{AB}$ is $2 \mathrm{~kb}$, megaprimer AM1 can be up to $\sim 1.5-\mathrm{kb}$ long, since $2 \mathrm{~kb}$ and $1.5 \mathrm{~kb}$ can be separated reasonably well in agarose gels. However, if the gene is $8 \mathrm{~kb}$, the megaprimer should not be bigger than, say, $7 \mathrm{~kb}$, since 8- and 7-kb fragments would migrate so close to each other.)

2. When the mutation is to be created near $B$, make an $M$ primer of the opposite polarity, e.g., M2 and synthesize BM2 megaprimer (rather than AM2). See Fig. 1.

3. When the mutation is at or very near the $5^{\prime}$ or $3^{\prime}$ end of the gene (say, within 1-50 nucleotides), there is no need to use the megaprimer method! One can simply incorporate the mutation in either A or B primer and do a straightforward PCR. In borderline situations, such as when the mutation is, say, 120 nucleotides away from the 5' end of the gene, incorporation of the mutation in primer $A$ may make the primer too big to synthesize; or else, it will make the megaprimer AM1 too short to handle conveniently. In such a case, simply back up primer A a few hundred bases further into the vector sequence in order to make AM1 megaprimer longer. In general, remember that primers A and B can be located anywhere on either side of the mutant region, and try to utilize that flexibility as an advantage when designing these primers.

4. In addition to the standard rules of primer design described in Chapter 2 (such as, a near 50\% GC content, sequence specificity, extra "clamp" sequence for restriction, absence of self-complementarity, and so forth), attention should be paid to the following aspects. As stated before, primers $A$ and $B$ should contain unique restriction sites for ease of cloning. As regards the $M$ primer, two things are important. First, the muta- 
tional mismatch should not be too close to the $3^{\prime}$ end of the primer. Mismatch at the very $3^{\prime}$ nucleotide of the primer will virtually abolish amplification by Taq polymerase. For best results, the mismatch should be at least four bases away from the $3^{\prime}$ end of the primer. Second, as described in detail in Note 1 , the $5^{\prime}$ end of the $M$ primer should preferably be located such that there is at least one (two or more is better) $T$ residue in the template strand of the same sense just upstream of this end of the primer. If a $T$ is not available, try to have the "wobble" base of a codon just upstream of the 5 ' end of the $M$ primer so that substitution of this base with A will code for the same amino acid (see Note 1).

\subsection{PCR 1: Synthesis of Megaprimer}

1. Assemble a standard $100-\mu \mathrm{L}$ PCR reaction (see Chapter 1 ) containing 5-50 ng of template DNA (containing the cloned gene) and $0.2-0.4 \mu \mathrm{g}$ each of primers $A$ and $\mathrm{Ml}$ (assuming a primer length of 25 nucleotides).

2. Amplify by PCR using the following cycle profiles (see Notes 2 and 3 ):

Initial denaturation

30-35 main cycles

Final extension

To achieve a better yield of megaprimer, do four such PCRs, $100 \mu \mathrm{L}$ each, in separate tubes, as described.

3. Remove oil overlay as follows. Freeze PCR tubes, then thaw just enough so that the oil overlay melts but the aqueous reaction stays frozen. Remove as much oil as possible. Then use a drawn-out round tip to transfer $\sim 80 \mu \mathrm{L}$ of the lower, aqueous layer carefully to a clean Eppendorf tube, wiping the outside of the tip to remove any adhering oil. It is important to remove the oil completely, otherwise, the sample will float up when loaded in horizontal agarose gels in step 4 (this section). Pool about $320 \mu \mathrm{L}$ from four reactions. Reduce volume to $\sim 40$ $\mu \mathrm{L}$ in SpeedVac (Savant Instruments, Farmingdale, NY) concentrator (or other means of evaporation or lyophilization).

4. Gel-purify megaprimer in standard agarose gels made in TBE in presence of ethidium bromide (see Chapter 1). Load in two or three wells in a midsize horizontal gel. Use an appropriate concentration of agarose $(0.7-1.2 \%)$ depending on the length of the megaprimer product to be purified (see Note 4). Perform electrophoresis until a good separation of the megaprimer and the small primer has been achieved.

5. Locate the megaprimer band by UV light, cut out the gel slices, place all slices of the same megaprimer in one slot of the electroelutor that is 
already filled with TE buffer or TBE buffer (see instruction manual of the electroelutor). Make sure that the apparatus is leveled, that buffers in the two chambers are connected, and that all buffer flow has stopped. Clear out any air bubble that may be trapped in the V-shaped grooves and put $75 \mu \mathrm{L}$ of $8 M$ ammonium acetate containing $0.01 \%$ BPB in each groove. Electrophorese at $150 \mathrm{~V}$ for required period (approx 15-30 min), as judged by the disappearance of the DNA band from the gel slice into the V groove (monitored by a hand-held UV light). Do not disturb the apparatus during elution.

6. Discontinue electrophoresis, carefully drain buffer out of both chambers, collect $400 \mu \mathrm{L}$ of ammonium acetate solution containing DNA from each $\mathrm{V}$ groove and transfer into a sterile clean microcentrifuge tube. Add 2-4 $\mu \mathrm{g}$ of carrier tRNA at this point to improve recovery. See Notes 5 and 6.

7. Clean the DNA by the usual phenol-chloroform extraction (see Chapter 1), precipitate with $2.5 \mathrm{vol}(1 \mathrm{~mL})$ of $95 \%$ ethanol (do not add extra salt), followed by centrifugation at maximum speed in a microcentrifuge for $15 \mathrm{~min}$.

8. Wash DNA pellet with prechilled $70 \%$ ethanol and dissolve in $20 \mu \mathrm{L}$ of sterile water. Expect 30-60\% recovery of DNA irrespective of size.

\subsection{PCR 2}

1. Assemble a second PCR reaction with $100 \mu \mathrm{L}$ final vol containing $1-2 \mu \mathrm{g}$ (note the higher than usual amount, also see Note 5) of plasmid template (the same template that was used in Section 3.2., step 2 ), $0.2-0.4 \mu \mathrm{g}$ primer B, 20-30 pmol of gel-purified megaprimer (3-5 $\mu \mathrm{g}$ for a 250-bp DNA), and standard concentration of buffer and nucleotides (see Chapter 1). Generally, expect to use up all the megaprimer recovered in step 4 in this PCR in order to achieve a good yield of the product! Use the maximum allowable temperature for annealing in the thermal cycle, as dictated by the smaller primer $\mathrm{B}$. Ignore the megaprimer for annealing considerations, since its $T_{\mathrm{m}}$ will be too high for the smaller primer.

2. Amplify by PCR using the cycle profiles described in step 2 of Section 3.2. using 25 main cycles.

3. Purify the mutant PCR product as described in Section 3.2., steps 4-7. It is now ready for restriction, ligation, and so forth by the use of standard procedures. See Notes 8 and 9.

\section{Notes}

1. The problem of nontemplated insertions and its solution will be published in detail elsewhere (Barik, manuscript in preparation). In brief, Taq polymerase has a tendency to incorporate nontemplated residues, 
particularly $\mathrm{A}$, at the $3^{\prime}$ end of the daughter polynucleotide strand at a certain frequency (3). These are then copied and amplified into the double-stranded product. This is generally not a problem in standard PCR where the termini of the product are usually cleaved off by restriction enzymes for cloning purposes. However, in the megaprimer method, the whole megaprimer is directly incorporated into the final product. Therefore, nontemplated A residues in the megaprimer will eventually show up in a certain percentage of the final product and cause a mutation that may be undesirable. The frequency of such "error" is usually low and megaprimers with a mismatch at the 3 ' end will not prime well; however, the frequency may be appreciable in some cases (Barik, manuscript in preparation). There are two kinds of solutions to this problem; one kind, exemplified by $a$ and $b$ below, does not introduce the nontemplated base or removes it; the other kind, described in c, does not remove the nontemplated base but tolerates the alteration.

a. Use the thermostable Vent DNA polymerase (New England Biolabs, Beverly, MA), which has a 3 ' exonuclease activity; follow the manufacturer's recipe. A typical 100- $\mu \mathrm{L}$ PCR will contain: $20 \mathrm{mM}$ Tris- $\mathrm{HCl}, \mathrm{pH} 8.8$ (at $25^{\circ} \mathrm{C}$ ), $10 \mathrm{mM} \mathrm{KCl}, 10 \mathrm{mM}\left(\mathrm{NH}_{4}\right)_{2} \mathrm{SO}_{4}, 2 \mathrm{mM}$ $\mathrm{MgCl}_{2}, 0.1 \%$ Triton $\mathrm{X}-100,100 \mu \mathrm{g} / \mathrm{mL}$ acetylated BSA, $200 \mu M$ of each dNTPs, 2 U of Vent polymerase, and standard amounts of template and primers. The temperature and time values of the thermal cycles are identical to those for Taq polymerase. Vent polymerase is a relatively recent product and may need some optimization.

b. Treat the purified megaprimer with a DNA polymerase that has a $3^{\prime} \rightarrow 5^{\prime}$ exonuclease activity. Following the first round of PCR reaction, remove oil as described in step 3 of Method. Add DTT to a final concentration of $1 \mathrm{mM}$ (even if the PCR buffer had DTT). Add $2.5 \mathrm{U}$ of T4 DNA polymerase or $7 \mathrm{U}$ of Klenow enzyme (4). Incubate at $37^{\circ} \mathrm{C}$ for $5 \mathrm{~min}$ with the $\mathrm{T} 4$ enzyme or at $15^{\circ} \mathrm{C}$ for $30 \mathrm{~min}$ with Klenow. Inactivate the polymerases by heating at $65-70^{\circ} \mathrm{C}$ for $5-10$ min and then proceed to purify the megaprimer after reducing volume, gel-purifying, and so forth as in step 4 of Method. Since all exonuclease digestions are hard to control, this method is not highly recommended and should be used as a last resort. Instead try the following method.

c. Tolerate the alteration (If you can't beat 'em, join 'em): This is the method of choice. It relies on clever primer design and does not require any extra step (in preparation). There are two ways of achieving this. As an example, suppose the relevant region of the wild-type sequence is (the amino acids are shown at the bottom in single-letter codes): 


\section{5'- AAA CTG CCA ACT CCG TCA TAT CTG CAG -3'}

$\begin{array}{rllllllll}3 & - \text { TTT GAC } \\ \text { K } & \text { L } & \text { P } & \text { T } & \text { P } & \text { S } & \text { Y } & \text { L } & \text { Q }\end{array}$

and the $\operatorname{Ser}$ (TCA) is to be mutated to Ala (GCA). A mutant primer in the message sense (like $M 2$ in Fig. 1) may have the sequence ${ }^{5} \mathrm{CA}$ ACT CCG GCA TAT CTG CAG ${ }^{3}$ (the boldface $G$ being the mutant base). However, when this M2 primer and primer B is used in PCR, the nontemplated $A$ incorporated at the $M 2$ end of the product (megaprimer) will result in the sequence:

\section{5'TCA ACT CCG GCA TAT CTG CAG - \\ 3'AGT TGA GGC CGT ATA GAC GTC -}

(the nontemplated $\mathrm{A} / \mathrm{T}$ is italicized). When incorporated into the final product, this megaprimer will produce the following mutant (the italicized amino acids are altered from the wild-type sequence):

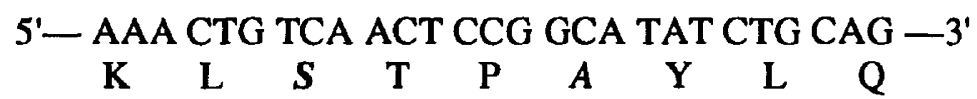

resulting in an undesired Ala $\rightarrow$ Ser change (boldface). To avoid this, make the following M2 primer: ${ }^{5} \mathrm{G}$ CCA ACT CCG GCA TAT CTG CAG ${ }^{3}$ so that there is a $T$ residue upstream of the 5 ' end of M2 in the template sequence; any extratemplated $T$ in this strand of the megaprimer will therefore match with the $T$ residue in the wild type sequence and will not cause any mutation.

When no $T$ residues are available, use the wobble base of a codon. This is possible when the primary purpose of the clone is to produce a protein product; thus, substitution of a codon with another, synonymous codon is permissible (make sure that the resultant change in the nucleotide sequence is acceptable in terms of introduction or loss of restriction sites, and so forth). Now, make the following M2 primer: ${ }^{5}$ ACT CCG GCA TAT CTG CAG ${ }^{3}$, so that the codon upstream of it is CCA. The nontemplated $T$ will change this codon to CCT; however, since they both code for proline, the protein will remain unaltered.

2. As a rule, elongation time (at $72^{\circ} \mathrm{C}$ ) in a PCR cycle should be proportional to the length of the product. An approximate guideline is $1 \mathrm{~min}$ of elongation $/ \mathbf{k b}$, i.e., 100 nucleotides $=10 \mathrm{~s} ; 500$ nucleotides $=40 \mathrm{~s} ; 1$ $\mathrm{kb}=1 \mathrm{~min} 40 \mathrm{~s} ; 2 \mathrm{~kb}=3 \mathrm{~min}$, and so on.

3. Annealing temperature is primarily governed by the base composition of the primers. A golden rule is to calculate the $T_{\mathrm{m}}$ of the primer as 
follows: Add $2^{\circ} \mathrm{C}$ for each $\mathrm{A}$ or $\mathrm{T}$, and $4^{\circ} \mathrm{C}$ for each $\mathrm{G}$ or $\mathrm{C}$, then deduct $4^{\circ}$. Example: for a 22-nucleotide primer with $10 \mathrm{G}+\mathrm{C}$ and $12 \mathrm{~A}+\mathrm{T}, T_{\mathrm{m}}$ is $[(10 \times 4+12 \times 2)]=64^{\circ} \mathrm{C}$; therefore, anneal at $60^{\circ} \mathrm{C}$. However, the upper limit of the annealing temperature for any primer is $72^{\circ} \mathrm{C}$, since it is the elongation temperature of the (Taq) polymerase.

4. Use $0.8-1.2 \%$ agarose for products ranging from $100-300 \mathrm{bp} ; 0.7 \%$ for $0.5-1 \mathrm{~kb} ; 0.6-0.7 \%$ for $1 \mathrm{~kb}$ and higher, and so forth. For making megaprimers in the 100-300-bp size range, it is important that the template preparation does not contain too much tRNA, which otherwise tend to mask the megaprimer in agarose gel and make visualization and purification of the megaprimer difficult.

5. If in the second round of PCR, higher concentration of template still does not help (very rare), it is advisable to make a single-stranded megaprimer (the priming strand, Fig. 1) by using the technique of "asymmetric" PCR in the first round. This is done by using a limiting amount of primer M1 (20-40-fold less, i.e., 5 pmol) while using the standard amount of primer A $(\sim 0.3 \mathrm{mg})$ in an otherwise identical PCR. A smaller number of cycles ( 20 cycles) and a slightly lower annealing temperature $\left(50-55^{\circ} \mathrm{C}\right)$ are recommended (5). The major problem in singlestranded DNA (ssDNA) PCR is the difficulty in locating the ssDNA in the gel: it does not bind EtBr well, migrates faster than the its doublestranded counterpart, and produces diffused or multiple bands. However, once standardized with a particular ssDNA product, the procedure can be used routinely for the same DNA. Note that it will be difficult to remove extratemplated nucleotides, if any, from ssDNA megaprimers; thus, plan $\mathrm{c}$ in Note 1 should be used in conjunction.

6. The electroelution procedure for purification of PCR products described here has been chosen as the single, most versatile method for such purpose. Several workers may prefer other methods for more specialized and routine applications, a few of which are as follows:

a. Centricon-30 spin filtration column (Amicon) may be used directly with the PCR reaction to separate the megaprimer product from the small PCR primers provided the megaprimer is sufficiently bigger than the smaller primers. Megaprimer purified by this method will also contain the template; however, this is of no concern since the same template is going to be used in the second round of PCR. Follow the recommended procedure (6).

b. Freeze-squeeze method (7): The method works best for fragments $<500 \mathrm{bp}$. The agarose gel slice containing the DNA fragment is taken in an Eppendorf tube, frozen in a dry ice-ethanol bath $(10 \mathrm{~min})$ or in a $-70^{\circ} \mathrm{C}$ freezer $(20 \mathrm{~min})$ and then spun at room temperature in a 
microcentrifuge for $15 \mathrm{~min}$. The recovered (30-70\%) megaprimer DNA can be used directly in PCR.

c. The GeneClean method (Bio 101, La Jolla, CA) utilizes the property of DNA to bind to glass (specially prepared, fine glass powder is supplied in the kit), however, it works best for DNA segments $>500$ bp. It is a rather elaborate procedure and is outside the scope of this chapter. The reader must follow the detailed instructions that come with the kit. A somewhat modified version has been published (1).

7. In a recent method (8), DNA fragments in low-melting-point agarose slices have been directly used in PCR reaction apparently without any problem. Since a good quantity of the megaprimer is important for the second PCR, it might be worth trying to use it in a similar manner. This will bypass the need to recover it from the gel. Further optimization of the method is advised.

8. As in any cloning procedure, the final mutants obtained by the megaprimer method must be confirmed by DNA sequencing. This can be done either by directly sequencing the PCR product (Chapters 13 and 14) or after cloning the mutant product in plasmid vectors. When using the dideoxy method, PCR primer A or B can be used as sequencing primers as well.

9. Other methods of site-specific mutagenesis exist that are based on PCR. These are discussed in detail in Chapters 24-27.

\section{References}

1. Sarkar, G. and Sommer, S. S. (1990) The "megaprimer" method of site-directed mutagenesis. BioTechnıques 8, 404-407.

2. Barik, S. and Galinski, M. (1991) "Megaprimer" method of PCR: Increased template concentration improves yield. BioTechniques 10, 489,490.

3. Clark, J. M. (1988) Novel non-templated nucleotide addition reactions catalyzed by procaryotic and eucaryotic DNA polymerases. Nucleic Actds Res. 16, 9677-9686.

4. Maniatis, T., Fritsch, E. F., and Sambrook, J. (1982) Molecular Cloning: A Laboratory Manual. Cold Spring Harbor Laboratory, Cold Spring Harbor, NY, pp. 113-119.

5. Finckh, U., Lingenfelter, P. A., and Myerson, D. (1991) Producing singlestranded DNA probes with the Taq DNA polymerase: A high yield protocol. BıoTechniques 10, 35-39.

6. Higuchi, R., Krummel, B., and Saiki, R. K. (1988) A general method of in vitro preparation and specific mutagenesis of DNA fragments: Studies of protein and DNA interactions. Nucleic Acids Res. 16, 7351-7367.

7. Stoflet, E. S., Koeberl, D. D., Sarkar, G., and Sommer, S. S. (1988) Genomic amplification with transcript sequencing. Science 239, 491-494.

8. Zintz, C. B. and Beebe, D. C. (1991) Rapid re-amplification of PCR products purified in low melting point agarose gels. BioTechnıques 11, 158-162. 